\title{
End-to-end data acquisition pipeline for the Cherenkov Telescope Array
}

\author{
E. Lyard*, R. Walter for the CTA Consortium \\ ISDC, University of Geneva, Switzerland \\ E-mail: etienne.lyard@unige.ch roland.walter@unige.ch
}

\begin{abstract}
The Cherenkov Telescope Array (CTA) will operate several types of telescopes and cameras. The individual camera trigger rates will vary much - from 0.6 to $15 \mathrm{kHz}$ - while the content of the raw data will be heterogeneous. Raw data streams of up to $43 \mathrm{Gbps}$ per telescope must be handled efficiently, from the camera front-ends down to the on-site repository and real-time analysis. In addition, the system must transcode all raw data to a common, pre-calibrated format.

We will present the pipeline that we propose to implement this data acquisition pipeline. It will format the raw data to a common structure, provide facilities to run camera-specific algorithms and compress and write data to the on-site repository. We will also present the Python interface that allows the analysis pipeline to access the data. Eventually, the two strategies foreseen to interface the camera servers will be detailed and the current status of the developments for CTA will be given, with the last performance figures measured.
\end{abstract}

35th International Cosmic Ray Conference - ICRC2017

10-20 July, 2017

Bexco, Busan, Korea

${ }^{*}$ Speaker. 


\section{Introduction}

The Cherenkov Telescope Array (CTA) [1] will operate more than 100 telescopes of different sizes between 4 and 23 meters in diameter. Each type of telescope will have one or two different kinds of Cherenkov camera, leading to very heterogenous data outputted by the telescopes. This document presents the pipeline that we propose to deploy to handle these data along with performance figures obtained from the prototype implementation of it.

The pipeline itself is composed of several modules as seen on figure 1 and it is the evolution of the prototyping activities presented at ICRC2015 [2]. The camera server interface allows the pipeline to readout event data from the camera servers. It comes in two flavours: native and bridged. The native interface delivers the unified format while the bridged version has a separate component (or bridge) that transcodes the camera native format to the unified one. The parameter extraction module pre-calibrates the events and extracts their high-level parameters, such as Hillas parameters. This is done early in the pipeline to help reduce the overall throughput of the system. The array event builder retrieves event parameters and assembles them into complete array events that are then forwarded to the real-time analysis. Due to the high trigger rate of the array (between 30 and $50 \mathrm{kHz}$ ), array event building will be load-balanced across several physical nodes. The Repository Writer receives raw, unified event data from a single telescope, applies a compression algorithm and writes the data to the on-site repository. The current prototype uses the ZFITS file format [3] and custom compression, but the module is flexible enough to allow other file formats and compressions to be used.

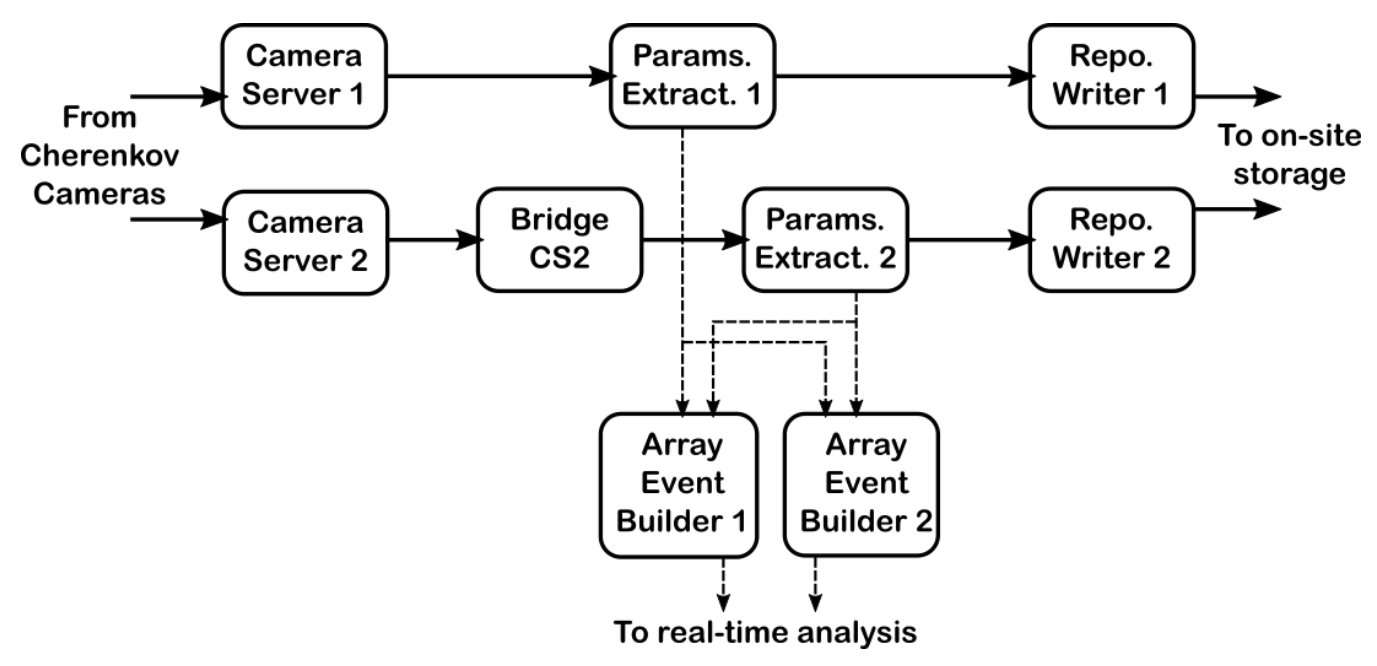

Figure 1: Overview of a data acquisition pipeline (DAQ) architecture with two telescopes. Solid lines denote raw event data while the dashed lines correspond to event parameters. Camera server 1 has a native interface to the DAQ while camera server 2 has a bridged interface. Raw event data are compressed and written to the on-site repository, while extracted event parameters are forwarded to array event builders.

Note that the selection of events based on stereo triggers is done entirely by the camera servers and thus not included in this pipeline. 
The pipeline runs inside the Alma Common Software framework (ACS) [4] and makes heavy use of ZeroMQ (ZMQ) [5] and of the Google protocol buffers [6]. It is modular and allows modules to be instantiated as stand-alone executables or ACS component, or distributed across several compute nodes. Load-balancing is built-in and thus virtually any data rate can be managed as long as the on-site infrastructure can handle it.

The pipeline comes with a raw-events reader class written in $\mathrm{C}++$. This reader has been interfaced with ctapipe [7] so that the ZFITS format can be used readily to analyse the data from early telescopes.

\section{Camera server readout}

Camera interfacing is done in two different ways: native and bridged. In the native interface the camera teams use the API provided by the DAQ pipeline to ship complete events downstream. Provided that the hardware is fast enough, one output stream delivers a throughput of approximately $9 \mathrm{Gbps}$ to a $10 \mathrm{Gbps}$ Ethernet interface. The CPU usage is in the order of 1.5 cores. Using infiniband and ip-over-ib instead of Ethernet suggests that better performances are to be expected, in the order of $12 \mathrm{Gbps}$ per stream. The bridged interface was not investigated very deeply yet, but performances up to $7 \mathrm{Gbps}$ for a single stream were achieved while interfacing Flashcam [8]. This test was done using a dummy Flashcam camera server producing events and sending them downstream using the a custom library from the FlashCam project called TMIO. The bridge was receiving events via TMIO in the Flashcam native format and was transcoding them to the unified format in protocol buffers. Unified events were then sent to a ZMQ receiver.

\subsection{Integration to camera servers}

So far cameras servers used their own code and memory management to build complete single telescope events. Complete events are then copied to a protocol buffers data structure before being serialised to a ZMQ socket. This extra copy of the data in memory was avoided by the NectarCam team. Not only did they use the provided API to send events, but they also used it to allocate the memory used to buffer the data. This allows incoming event data to be put to the protocol buffer structure with no intermediate copy, thus avoiding this extra copy. This approach produced the best performances so far: $18 \mathrm{Gbps}$ using two parallel streams [9].

\subsection{LST readout}

It has been recently agreed that the maximum readout data rate for large size telescopes (LST) would be $24 \mathrm{Gbps}$. It is foreseen that the LST event builder will reuse the same software as for NectarCam, thus ensuring maximum integration of the interface to DAQ. However, the current DAQ software has only been verified up to $18 \mathrm{Gbps}$ and pushing the performances up to $24 \mathrm{Gbps}$ might be challenging. Indeed, as shown on figure 2, preliminary tests done with several $10 \mathrm{Gbps}$ interfaces indicate that the performances do not grow linearly if more interfaces / streams are added. This is more likely due to a suboptimal usage of the available resources, for example if non uniform memory access boundaries are crossed. The $24 \mathrm{Gbps}$ throughput from the requirement seems in our 
reach, however it may need 4 interfaces rather than 3 unless further optimisations are made in the software. This will be closely investigated in the coming months, and the most simple solution will be selected for the commissioning of LST1.

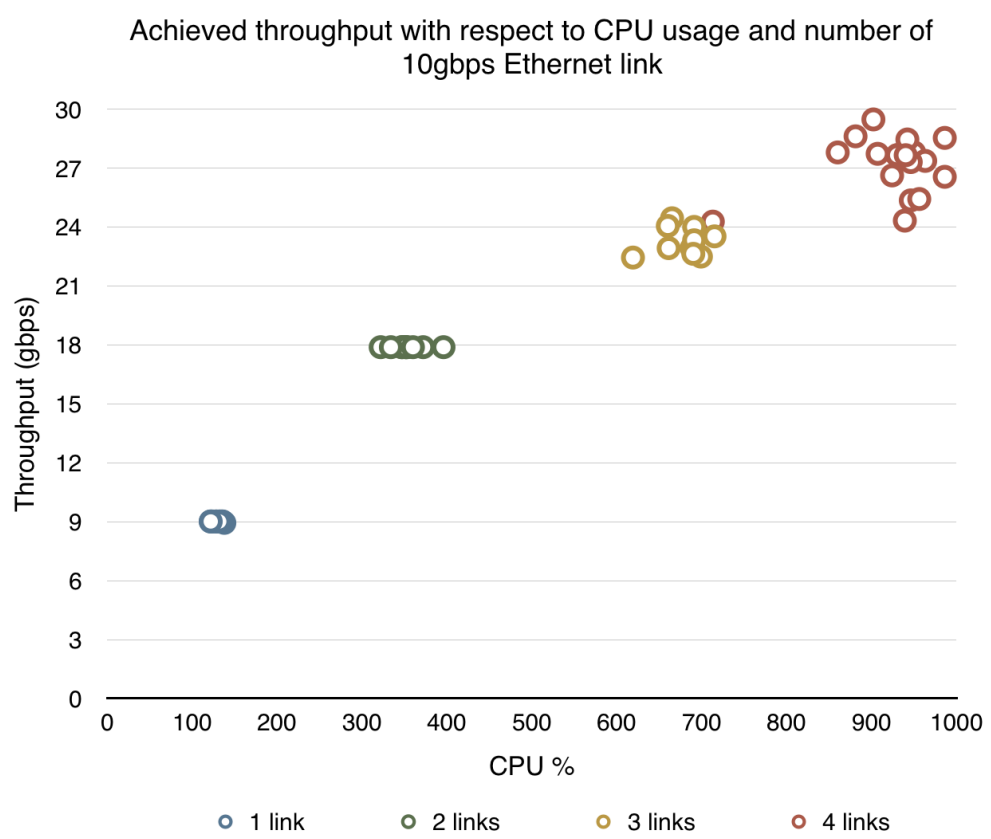

Figure 2: Throughput performances using 1,2, 3 and 4 times 10Gbps interfaces with $1 \mathrm{ZMQ}$ stream per interface. The test was sending 100 GBytes of event data to remote clients. There was $2 x$ more clients than data producers and the clients just discarded the data. The tests was repeated 10 times for 1 to 3 interfaces, and 20 times for 4 interfaces.

\section{Processing facilities}

The DAQ pipeline main task is to collect raw event data from the camera servers and to write it to the on-site repository. Besides this task, it will also run the parameter extraction and array-event reconstruction from these parameters.

\subsection{Parameter extraction}

The parameter extraction is located early in the pipeline to help reduce the overall throughput. Indeed, event parameters are much smaller in size compared to raw events and can be extracted for a single telescope. Sending event parameters to the array event builder rather than raw data makes the extra throughput negligible compared to the bulk data transfer.

The algorithms used to pre-calibrate the event data will come from the camera teams as they are the ones who know best how to calibrate their instrument. This algorithm will be reversible so that a better calibration can be applied by the offline analysis pipeline. The algorithms used to extract the event parameters will be common to all cameras and most likely run in python inside the ctapipe framework.

As of now, it remains unclear what will be the details of the interface between the DAQ pipeline 
and ctapipe. Our first choice is to use the protocol buffers and ZMQ once again to interface the DAQ component with a generic ctapipe component running in ACS. If better performances are needed, then the ctapipe routines will be called directly from the DAQ C++ component. However, as this approach is less flexible than our first choice, it will be implemented only if needed.

\subsection{Array event builder}

The array event builder is the interface between the DAQ pipeline and the real-time analysis. It collects single telescope image parameters and assembles them into array events based on the stereo trigger information. Due to the high trigger rate of CTA it is foreseen that more than one instance will be needed.

As there might be more than one parameter extraction instances in case of high throughput telescopes (e.g. LSTs), it is not trivial how to route each event parameter to its array building instance. Early prototyping activities made the array builder request a given event ID to all parameter extraction nodes. Only the node that had processed this event would in-turn forward the data to the requesting event builder.

This approach was successful during prototyping, but it was not implemented up to the scale of CTA. As soon as the final data rates for all telescopes will be known, along with the performances of the parameter extraction algorithms, then we will repeat the prototyping with a more realistic setup.

\section{Interface to the on-site repository}

The interface to the on-site repository is two-fold: read and write. The writing is done by a Repository Writer ACS component while the reading is done by interfacing a $\mathrm{C}++$ class to the ctapipe framework.

\subsection{Repository Writer}

We implemented the repository writer as an ACS C++ component. It listens to ZMQ streams, sorts incoming events and writes them in ZFITS format. The compression used in ZFITS is a custom scheme, yet to be finalised once real CTA data will be available. Various compression schemes were tried out so far, as depicted on figure 3. It seems obvious that a compromise has to be made between speed and compression ratio. Considering the long-term storage foreseen for CTA data it looks to us that it would be worth applying a specific compression algorithm per kind of data stored, as ZFITS allows to do.

\section{2 ctapipe reader}

A prototype implementation of a ZFITS reader for ctapipe was implemented. To avoid extra developments, it reuses the $\mathrm{C}++$ class from the DAQ pipeline instead of reading the data natively in python. The data are read and uncompressed by the $\mathrm{C}++$ code, serialised in protocol buffer format and given to python as a binary block. A lightweight python layer then decodes this binary block 


\begin{tabular}{|r|c|c|c|}
\hline $\begin{array}{r}\text { 100k events } \\
\text { version }\end{array}$ & $\begin{array}{c}\text { Compression } \\
\text { ratio }\end{array}$ & $\begin{array}{c}\text { Compression } \\
\text { time }\end{array}$ & $\begin{array}{c}\text { MB/s per } \\
\text { thread to } \\
\text { shm }\end{array}$ \\
\hline raw & 1 & 6.51 & 798.8 \\
\hline Izo & 1.35 & 25.54 & 203.6 \\
\hline split+lzo & 1.50 & 16.98 & 306.2 \\
\hline rice & 1.55 & 140.06 & 37.1 \\
\hline zlib & 2.05 & 952.51 & 5.5 \\
\hline split+zlib & 2.21 & 487.99 & 10.7 \\
\hline huffman16 & 2.33 & 44.36 & 117.2 \\
\hline fact & 2.37 & 51.01 & 101.9 \\
\hline diffthuffman16 & 2.44 & 49.80 & 104.4 \\
\hline specific & 2.89 & 42.62 & 122.0 \\
\hline & & & \\
\hline
\end{tabular}

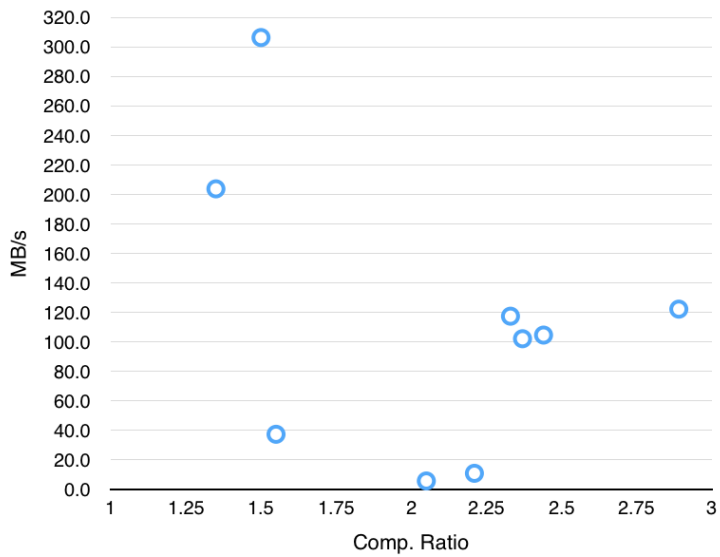

Figure 3: Compression ratios and throughput per core for various compression schemes. raw means that no compression was applied. lzo is the well-known real-time compression algorithm [10]. split means that the data are pre-processed by splitting the high and low part of the binary words. rice is the rice compression [11]. zlib uses the well-known gzip algorithm [12]. huffmanl6 is an implementation of the huffman coding on 16 bits [13]. fact is the scheme used by the FACT project to store their data [14]. diff means that the difference between each sample is stored rather than the samples themselves. specific means that the best performing algorithm on each type of data (indices, samples, ... ) was used for each column of the ZFITS file.

and delivers it to the expected ctapipe structure. A detailed view of the architecture of the reader can be seen on figure 4 .

\begin{tabular}{|c|c|c|c|c|c|c|c|c|c|c|}
\hline & \multicolumn{5}{|c|}{ protozfitsreader.cpp } & \multicolumn{5}{|c|}{ rawzfitsreader.py } \\
\hline ZFITS & $\underset{\text { Decompress }}{\stackrel{\text { Read }+}{\longrightarrow}}$ & $\begin{array}{c}\text { Internal } \\
\text { Binary }\end{array}$ & Convert to & $\begin{array}{l}\text { Protobuf } \\
\text { Events }\end{array}$ & Serialize & $\begin{array}{l}\text { Protobuf } \\
\text { Serial }\end{array}$ & De-serialize & $\begin{array}{l}\text { Protobuf } \\
\text { Events }\end{array}$ & \begin{tabular}{|c} 
Convert to \\
ctapipe
\end{tabular} & $\begin{array}{l}\text { ctapipe } \\
\text { Events }\end{array}$ \\
\hline
\end{tabular}

Figure 4: Architecture of the ZFITS reader prototype for ctapipe. The DAQ C++ class ProtoZFitsReader is used to perform the low level operations and obtain the events in a protocol buffer object. This object is then serialised and passed to the python side of the interface. The serial data are then de-serialised using the protocol buffers for python and converted to the data structure expected by ctapipe. This architecture has been easy to implement as only protocol buffer serial data are passed between $\mathrm{C}++$ and python. It is obviously suboptimal as extra format conversions take place. However, because most of the computation time is spent decompressing the data, the overhead remains small, in the order of 3 percent of the total time.

This approach had the advantage that it has been fast and effective to implement, thus allowing immediate usage by the early prototypes. The reader could be made more efficient if the interface between the $\mathrm{C}++$ class and python would be made natively rather than via the protocol buffers. Even though the overhead is very small, if ZFITS is selected as CTA raw data format then we will consider to make the effort to improve this interface.

\section{Future work}

The prototyping activities to interface remaining Cherenkov cameras is ongoing. As soon as 
the LST event builder becomes available we will perform the benchmark required to understand if the proposed architecture could work for such data rates.

In parallel, a prototype interface to the real-time analysis is being developed. We are currently focused in embedding the parameter extraction into the DAQ pipeline and will soon move to the updated version of the array event builder.

Once the data format for the raw data of CTA will be selected, we will make the required adjustments to the Repository Writer component.

\section{Acknowledgments}

This work was conducted in the context of the ACTL working group of the CTA Consortium. We gratefully acknowledge financial support from the agencies and organisations listed here: http://www.cta-observatory.org/consortium_acknowledgments

\section{References}

[1] B.S. Acharya et al., Introducing the CTA concept., Astroparticle Physics, Volume 43, 2013, Pages 3-18, ISSN 0927-6505, http://dx.doi.org/10.1016/j.astropartphys.2013.01.007.

[2] E. Lyard et al. Modern middleware for the data acquisition of the Cherenkov Telescope Array. In: vol. ICRC2015. 2015-08. arXiv: 1508.06473 [astro-ph.IM]

[3] W. Pence et al. A Tiled-Table Convention for Compressing FITS Binary Tables., ArXiv e-printsarXiv:1201.1340.

[4] G. Chiozzi et al., The ALMA common software: a developer-friendly CORBA-based framework. in Advanced Software, Control, and Communication Systems for Astronomy, Society of Photo-Optical Instrumentation Engineers (SPIE) Conference Series 5496, 205-218, 2004

[5] Hintjens, P., ZeroMQ: Code Connected., http://zeromq.org, 2014

[6] Google Inc., Protocol Buffers, http://developers.google.com/protocol-buffers, 2014

[7] Kosack, K., ctapipe: Low-level data processing pipeline prototype, https://github.com/cta-observatory/ctapipe, 2017

[8] G. Puehlhofer et al. FlashCam: a fully-digital camera for the medium-sized telescopes of the Cherenkov Telescope Array. In: vol. ICRC2015.2015-08. arXiv:1509.02434 [astro-ph.IM]

[9] D. Hoffmann and J. Houles, 40-Gbps data-acquisition system for NectarCAM CHEP 2016 Conference, 2016.

[10] Lempel-Ziv-Oberhumer(LZO) https://en.wikipedia.org/wiki/Lempel-Ziv-Oberhumer, retrieved June 2017

[11] R. F. Rice et al., Algorithms for high-speed universal noiseless coding., in: 9th AIAA Computing in Aerospace Conference, 1993. doi:10.2514/6.1993-4541.

[12] P. W. Katz, String searcher, and compressor using same, US Patent num. 5051745 (09 1991).

[13] D. Huffman, A method for the construction of MinimumRedundancy codes., Proceedings of the IRE 40 (9) (1952) 1098-1101. doi:10.1109/jrproc.1952.273898 
[14] M. L. Ahnen et al. Data compression for the First G-APD Cherenkov Telescope, Astronomy and Computing 12 (2015), pp. 191-199. issn: 2213-1337. 